\title{
INTOXICAÇÕES EXÓGENAS NA FAIXA ETÁRIA PEDIÁTRICA NO ESTADO DO MATO GROSSO:A ALTA PREVALÊNCIA DAS TENTATIVAS DE SUICÍDIO
}

\section{Autores \\ Erika Natielli Moreira Frizon ${ }^{1}$ Gabriela Coelho de Oliveira Luciana Mathias de Camargo Rauane Beatriz Wachholz ${ }^{1}$ Emmanuela Bortoletto Santos dos Reis ${ }^{2}$ Hugo Dias Hoffman Santos ${ }^{2}$ Rosa Maria Elias ${ }^{2}$ Thaís Caroline Dallabona Dombroski}

1. Discente do Curso de Medicina do Centro Universitário de Várzea Grande - UNIVAG

2. Docente do curso de Medicina do Centro Universitário de Várzea Grande - UNIVAG

\section{RESUMO}

Objetivo: Descrever o perfil das intoxicações exógenas de indivíduos da faixa etária pediátrica (0 a 19 anos) notificadas pelo Sistema de Informação deAgravos de Notificação (SINAN) no período de 2017a 2019 no estado de Mato Grosso. Metodologia: Estudo ecológico transversal efetuado através de dados procedentes do SINAN obtidos por meio do repositório DwWeb (SES-MT), durante o período de janeiro de 2017 a dezembro de 2019. Resultados: Foram registradas 236 notificações devido intoxicação exógena, sendo os medicamentos os principais responsáveis, com destaque para a classe dos benzodiazepínicos e antidepressivos; a maioria das notificações ocorreu na faixa etária de 15 a 19 anos (47,88\%), destas a principal circunstância de exposição ao agente tóxico foi a tentativa de suicídio. Conclusão: Conclui-se que as intoxicações exógenas por medicamentos apresentam maior prevalência nos adolescentes, os quais possuem motivação intencional, seguido das crianças menores de 10 anos devido aos acidentes com agentes químicos.

Palavras-Chave: Acidentes Domésticos; Tentativa de Suicídio; Envenenamento; Notificação Compulsória; Crianças.

\section{ABSTRACT}

Objective: The purpose of this paper is to describe the profile of the SINAN (Notifiable Diseases Information System) notified cases of exogenous intoxication, affecting the pediatric population (between 0 and 19 years old) of Mato Grosso, over the period from 2017 to 2019. Methodology: A cross-sectional ecological study was carried out using data provided by the DwWeb SES-MT (SINAN's supervisory system), collected over the period from January 2017 to December 2019. Results: There were 236 exogenous intoxication notified cases, major contributors being medicaments, mainly the benzodiazepines and the 
1. Vale NB - Centenário da Raquianestesia Cirúrgica. Rev Bras Anestesiol, 1998; 48: 507-520.

2. Choi PT, Galinski SE, Takeuchi L, et al. PDPH is a common complication of neuroaxial blockade in parturients: a meta-analysis of obstetrical studies. Can J Anaesth. 2003;50:460---9.

3. Turnbull DK, Shepherd DB. Post-dural puncture headache: pathogenesis, prevention and treatment. Br J Anaesth. 2003;91:718---29. antidepressants. Most notifications (47,88\%) occurred in the 15-19 year-old age group, being suicide attempts the main circumstance of exposure to the toxic agent. Conclusion: It can be concluded that exogenous intoxication caused by medicaments has the highest prevalence among teenagers, in non-accidental events, followed by children under the age of 10 , due to the accidents with chemical agents.

Keywords: Home and Leisure Accidents; Suicide Attempts; Poisoning; Mandatory Report; Children.

\section{INTRODUÇÃO}

A intoxicação exógena é o conjunto de efeitos nocivos decorrentes de alterações clínicas e/ou bioquímicas secundárias ao contato com um agente externo específico. Essa representa uma causa comum de morbidade na faixa etária pediátrica e ocorre de forma heterogênea, sofrendo influência de fatores intrínsecos ao indivíduo intoxicado como a idade, e fatores extrínsecos como o local de ocorrência. ${ }^{1,2}$

A faixa etária pediátrica faz parte do grupo de risco para as intoxicações exógenas devido características inatas das fases do desenvolvimento infantil, como a curiosidade natural das crianças e o hábito de colocar objetos na boca, e durante a adolescência há maior envolvimento nas intoxicações intencionais, seja por abuso do uso de drogas, ou até por tentativa de suicídio. ${ }^{1,3}$

Dentre as substâncias mais envolvidas nas intoxicações exógenas, destacam-se os agentes químicos e os medicamentos, tal dado se correlaciona com as principais circunstâncias de intoxicação, que são a forma acidental e a tentativa de suicídio.

A tentativa de suicídio ocorre quando a pessoa apresenta comportamentos autodestrutivos com intenção explícita ou implícita de autoextermínio, diversos métodos podem ser utilizados para tal objetivo, desde métodos violentos como enforcamento, automutilação, até método não violentos, como a ingestão de medicamentos ou substâncias sabidamente tóxicas. A ideação suicida ocorre quando o indivíduo apresenta pensamentos relacionados a comportamentos de 
2. Choi PT, Galinski SE, Takeuchi L, et al. PDPH is a common complication of neuroaxial blockade in parturients: a meta-analysis of obstetrical studies. Can J Anaesth. 2003;50:460---9.

4. Takmaz S, Ünal KC, Kaymak C, et al. Treatment of post-dural puncture headache with bilateral greater occipital nerve block. Headache. 2010;50:869-81. autoextermínio. ${ }^{4}$

A tentativa de suicídio se destacou como a principal causa de intoxicação exógena no estado de Mato Grosso na faixa etária pediátrica. Estudos epidemiológicos atuais, corroboram com este dado, demonstrando o grande aumento da incidência da tentativa de suicídio na faixa etária pediátrica.

Vários são os fatores que influenciam nas manifestações de quadros de intoxicações exógenas, bem como nas tentativas de suicídio, e ao compreender esses fatores é possível encontrar meios de prevenção e/ou redução de sua crescente incidência. ${ }^{2}$

Dessa forma, o objetivo desse estudo é identificar o padrão mato-grossense de ocorrências de intoxicações exógenas levando em consideração fatores importantes, tais como causa, faixa etária, agente e sexo; além de debater a relevância da tentativa de suicídio como seu desencadeante.

\section{MÉTODOS}

O presente trabalho trata-se de um estudo ecológico transversal, retrospectivo e descritivo, sobre as intoxicações exógenas entre crianças e adolescentes no estado de Mato Grosso no período de 2017 a 2019.

Os dados coletados para o estudo foram obtidos por meio do Repositório de dados dos Sistemas de Informação da Secretaria de Estado de Saúde de Mato Grosso (DwWeb). Analisaram-se 236 casos que ocorreram de janeiro de 2017 a dezembro de 2019, todos os casos notificados e confirmados de intoxicação exógena.

As variáveis analisadas foram: idade, sexo, local de exposição, município (cidades polos econômicos e número de habitantes), circunstância de exposição, evolução do caso, mês de notificação, agente tóxico, classe, acessibilidade, tentativas de suicídio. Quanto à acessibilidade, foi definido como fácil acesso, aquela substância que possui livre compra pela internet e, como difícil acesso a que necessita de receituários especiais ou comprovação de profissão para a compra. Os dados populacionais e econômicos foram obtidos pelo Instituto Brasileiro de Geografia e Estatística (IBGE) dos anos 2010 e 2017, 
respectivamente.

Elaborou-se uma tabela descritiva através do Microsoft Excel ${ }^{\circledR}$ e então, ela foi analisada pelo programa Epi Info versão 7 (Center for Diseases Control and Prevention) e, por fim, realizou- se sua transcrição.

\section{RESULTADOS}

Entre os anos de 2017 e 2019, 236 casos de intoxicação exógena entre indivíduos da faixa etária pediátrica (0 aos 19 anos) foram notificados no estado de Mato Grosso. Os medicamentos foram os principais responsáveis pelas intoxicações, totalizando 58,90\% dos casos (Figura 1), dentre estes as classes medicamentosas mais prevalentes foram os benzodiazepínicos (12,29\%), como clonazepam e diazepam, e os antidepressivos (9,32\%), como fluoxetina e amitriptilina. Dentre as substâncias não medicamentosas, os agentes químicos (16,10\%) foram os principais, sendo a água sanitária e o hidróxido de sódio, os mais envolvidos nas intoxicações (Tabelas 1 e 2).

Figura 1 - Comparação da porcentagem das substâncias medicamentosas e não medicamentosas envolvidas nas intoxicações exógenas.

\section{Uso de medicamentos nas intoxicações exógenas}

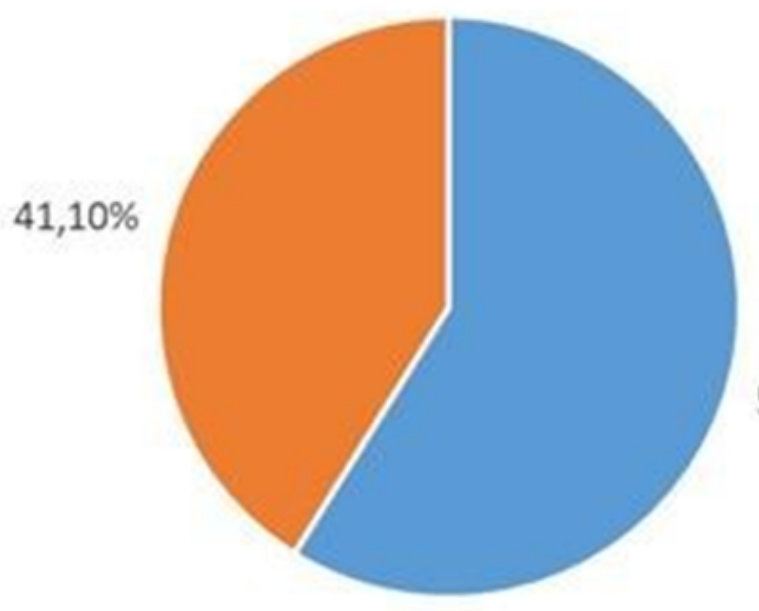

$58,90 \%$ 
Tabela 1 - Classificação das substâncias envolvidas nas intoxicações exógenas entre indivíduos da faixa etária pediátrica no Mato Grosso, nos anos de 2017 a 2019.

\begin{tabular}{|c|c|c|}
\hline Classe & $\mathbf{N}$ & $\%$ \\
\hline Agente químico & 38 & 16,10 \\
\hline Benzodiazepínico & 29 & 12,29 \\
\hline Antidepressivo & 22 & 9,32 \\
\hline Pesticida & 22 & 9,32 \\
\hline Herbicida & 11 & 4,66 \\
\hline Analgésico & 11 & 4,66 \\
\hline Antipsicótico & 12 & 5,08 \\
\hline Anti-inflamatório & 9 & 3,81 \\
\hline Raticida & 9 & 3,81 \\
\hline Anticonvulsivante & 8 & 3,39 \\
\hline Álcool & 7 & 2,97 \\
\hline Alimentos & 7 & 2,97 \\
\hline Antibiótico & 5 & 2,12 \\
\hline Antiemético & 5 & 2,12 \\
\hline $\begin{array}{l}\text { Anti-histamínico } \\
\text { Anticoncepcional }\end{array}$ & $\begin{array}{l}5 \\
3\end{array}$ & $\begin{array}{l}2,12 \\
1,27\end{array}$ \\
\hline Antifúngico & 3 & 1,27 \\
\hline Anti-hipertensivo & 3 & 1,27 \\
\hline Hipnóticos & 3 & 1,27 \\
\hline Não especificado & 3 & 1,27 \\
\hline Anticoagulante & 2 & 0,85 \\
\hline Anti-gripal & 2 & 0,85 \\
\hline Escabicida & 2 & 0,85 \\
\hline Estabilizador de humor & 2 & 0,85 \\
\hline Anfetamina & 1 & 0,42 \\
\hline Ansiolítico & 1 & 0,42 \\
\hline Antiparasitário & 1 & 0,42 \\
\hline Anti-parasitário & 1 & 0,42 \\
\hline Anti-séptico & 1 & 0,42 \\
\hline Antitérmico & 1 & 0,42 \\
\hline Anti-tireoidiano & 1 & 0,42 \\
\hline Ergotamina & 1 & 0,42 \\
\hline Fitoterápico & 1 & 0,42 \\
\hline Inibidor de Bomba & 1 & 0,42 \\
\hline Relaxante muscular & 1 & 0,42 \\
\hline Vasodilatador & 1 & 0,42 \\
\hline Vitaminas & 1 & 0,42 \\
\hline
\end{tabular}


Tabela 2 - Classificação dos agentes tóxicos envolvidos nas intoxicações exógenas entre indivíduos da faixa etária pediátrica no Mato Grosso, nos anos de 2017 a 2019.

\begin{tabular}{|c|c|c|c|c|}
\hline Classe da Substância & Agente tóxico & $\mathrm{N}$ & $\%$ do total & $\%$ da classe \\
\hline \multirow[t]{2}{*}{ Agente químico } & Água sanitária & $\begin{array}{l}1 \\
5\end{array}$ & 6,36 & 39,47 \\
\hline & Hidróxido de sódio & 4 & 1,69 & 10,53 \\
\hline \multirow[t]{3}{*}{ Benzodiazepínico } & Clonazepam & $\begin{array}{l}1 \\
4\end{array}$ & 5,93 & 48,27 \\
\hline & Diazepam & 3 & 1,27 & 10,34 \\
\hline & Fluoxetina & 1 & 4,24 & 45,45 \\
\hline \multirow[t]{2}{*}{ Antidepressivo } & Amitriptilina & $\begin{array}{l}0 \\
4\end{array}$ & 1,69 & 18.18 \\
\hline & Outros antidepressivos & 4 & 1,69 & 18,18 \\
\hline \multirow[t]{2}{*}{ Pesticida } & Cipermetrina & 9 & 3,81 & 40,90 \\
\hline & Metaldeido & 3 & 1,27 & 13,63 \\
\hline Herbicida & Glifosato & 4 & 1,69 & 26,67 \\
\hline \multirow[t]{2}{*}{ Analgésico } & Dipirona & 5 & 2,12 & 45,45 \\
\hline & Paracetamol & 5 & 2,12 & 45,45 \\
\hline Anti-inflamatório & Ibuprofeno & 3 & 1,27 & 33,33 \\
\hline \multirow[t]{2}{*}{ Antipsicótico } & Haloperidol & 4 & 1,69 & 44,44 \\
\hline & Risperidona & 3 & 1,27 & 33,33 \\
\hline \multirow[t]{2}{*}{ Raticida } & Bromadiolone & 4 & 1,69 & 44,44 \\
\hline & Brodifacoum & 3 & 1,27 & 33,33 \\
\hline \multirow[t]{2}{*}{ Anticonvulsivante } & Carbamazepina & 3 & 1,27 & 37,5 \\
\hline & Fenobarbital & 3 & 1,27 & 37,5 \\
\hline Álcool & Álcool/etanol não especificado & 5 & 2,12 & 71,43 \\
\hline Alimentos & Refrigerante & 3 & 1,27 & 42,86 \\
\hline Antibiótico & Amoxicilina & 2 & 0,85 & $40 \%$ \\
\hline Antiemético & Dimenidrinato & 2 & 0,85 & $40 \%$ \\
\hline Anti-histamínico & Cipraeptadina & 4 & 1,69 & $80 \%$ \\
\hline \multirow[t]{3}{*}{ Anticoncepcional } & Desogestrel & 1 & 0,42 & 33,33 \\
\hline & Desogestrel etinilestradiol & 1 & 0,42 & 33,33 \\
\hline & Etinilestradiol & 1 & 0,42 & 33,33 \\
\hline Antifüngico & Cetoconazol & 2 & 0,85 & 66,66 \\
\hline \multirow[t]{3}{*}{ Anti-hipertensivo } & Nifedipina & 1 & 0,42 & 33,33 \\
\hline & Atenolol & 1 & 0,42 & 33,33 \\
\hline & Metildopa & 1 & 0,42 & 33,33 \\
\hline Hipnóticos & Zolpidem & 3 & 1,27 & 100 \\
\hline
\end{tabular}


Quanto à circunstância de exposição, 107 casos notificados (45,34\%) foram descritos como decorrentes de tentativa de suicídio, sendo esta a principal causa de intoxicação identificada (Figura 2).

Dentre todas as faixas etárias, os indivíduos de 15 a 19 anos representaram a maioria dos envolvidos em intoxicações exógenas (Figura 3) totalizando 47,88\% dos casos, e, dentre esses, $73,45 \%$ ocorreram em decorrência da tentativa de suicídio.

Ademais, a tentativa de suicídio também foi a principal causa de intoxicação na faixa etária dos 10 aos 14 anos. Por outro lado, entre 0 a 9 anos, a principal circunstância de exposição ao agente tóxico foi acidental, a prevalência é ainda maior na faixa etária de 1 a 4 anos na qual 93,85\% das intoxicações ocorrem de forma acidental (Figura 4).

A exposição acidental é a segunda circunstância mais prevalente entre os casos estudados, correspondendo a $38,14 \%$ dos casos. Além das circunstâncias de exposição já citadas, também foram notificados casos em que a intoxicação ocorreu devido uso habitual $(5,51 \%)$, ingestão de bebida ou alimento (3,81\%), abuso (1,69\%), automedicação (1,69\%), erro de administração (1,69\%), entre outras (Figura 2).

Figura 2 -Porcentagem de casos de intoxicação exógena de acordo com suas circunstânciasde exposição.

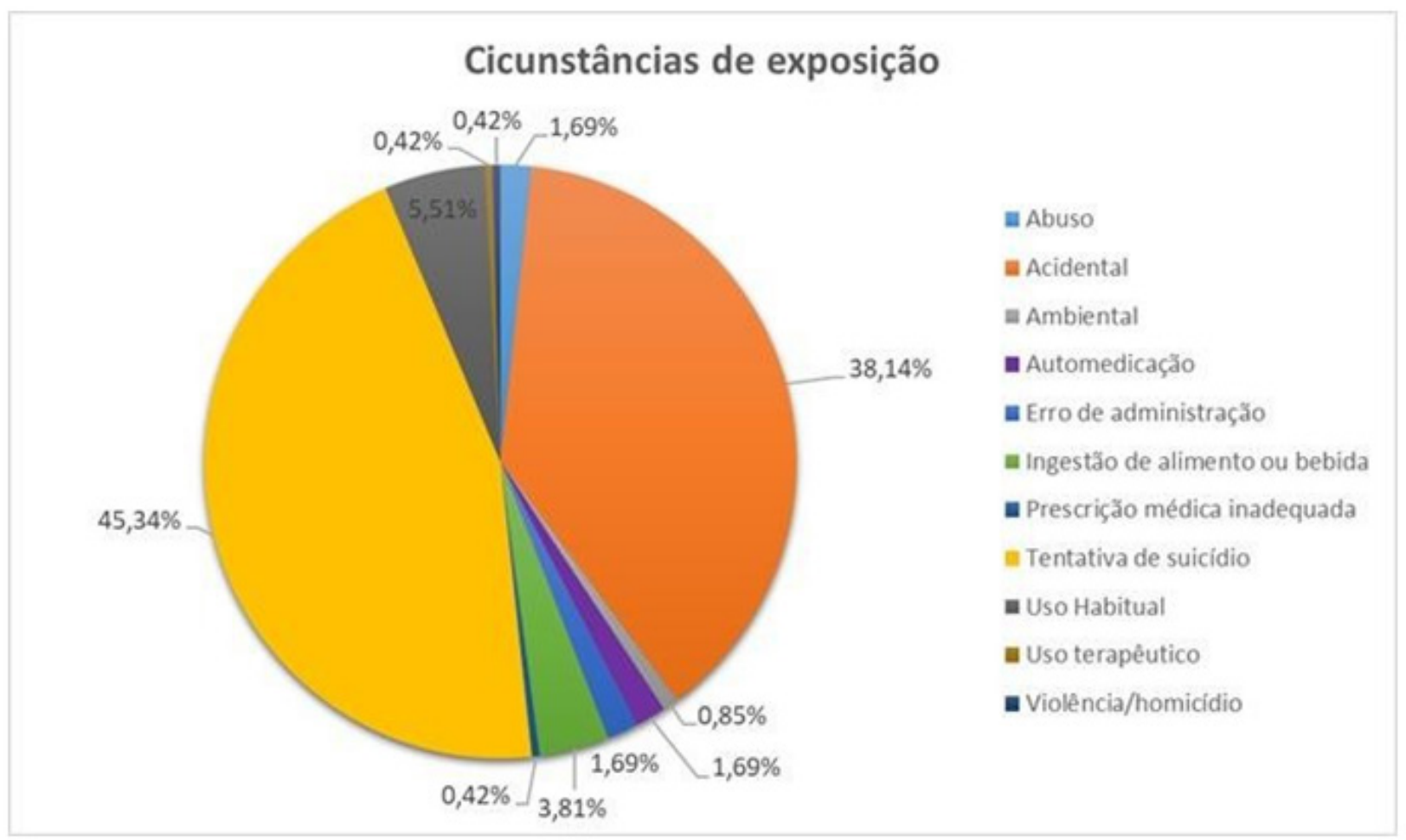


Figura 3 - Frequência das idades dos indivíduos que sofreram intoxicação exógena.

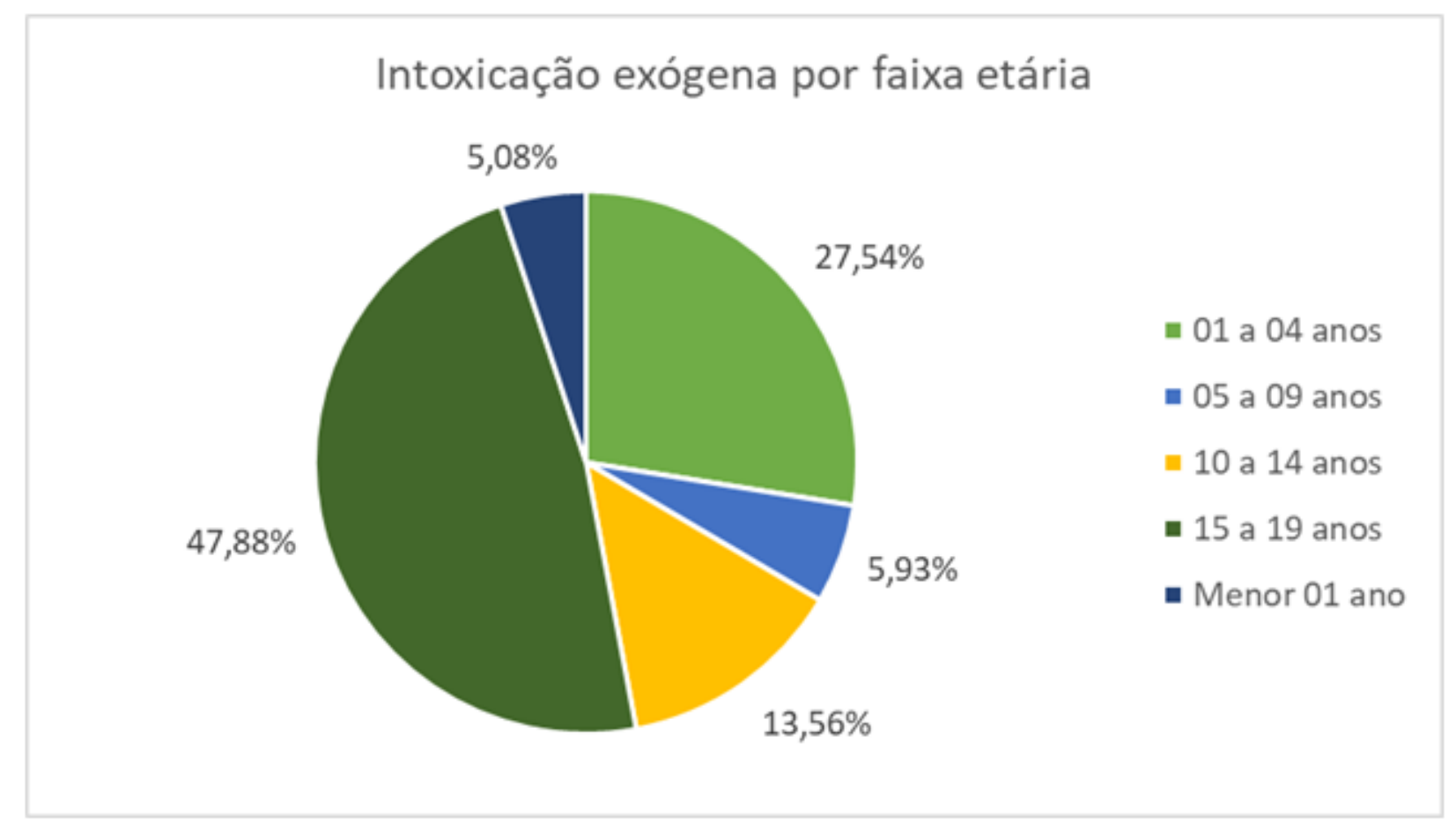

Figura 4 - Tentativa de suicídio de acordo com a faixa etária.

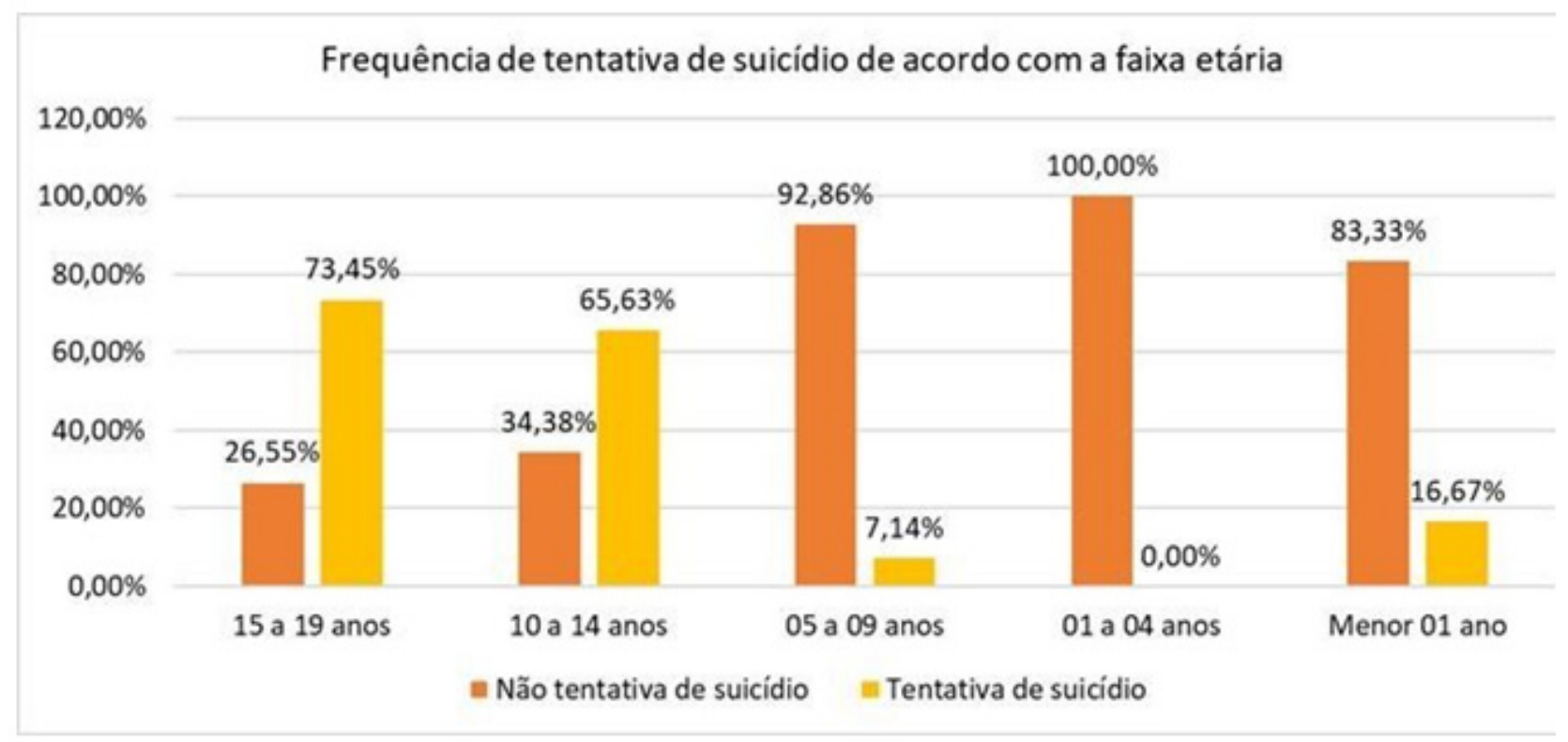

Quanto aos casos de tentativa de suicídio, verifica-se um aumento da incidência no período estudado, em 2017 a taxa de intoxicação exógena devido tentativa de suicídio foi de 15,89\%, já em 2018 e 2019 foi de $21,50 \%$ e 62,62\%, respectivamente 
(Figura 5).

Figura 5 - Porcentagem de tentativas de suicídio no período de 2017 a 2019.

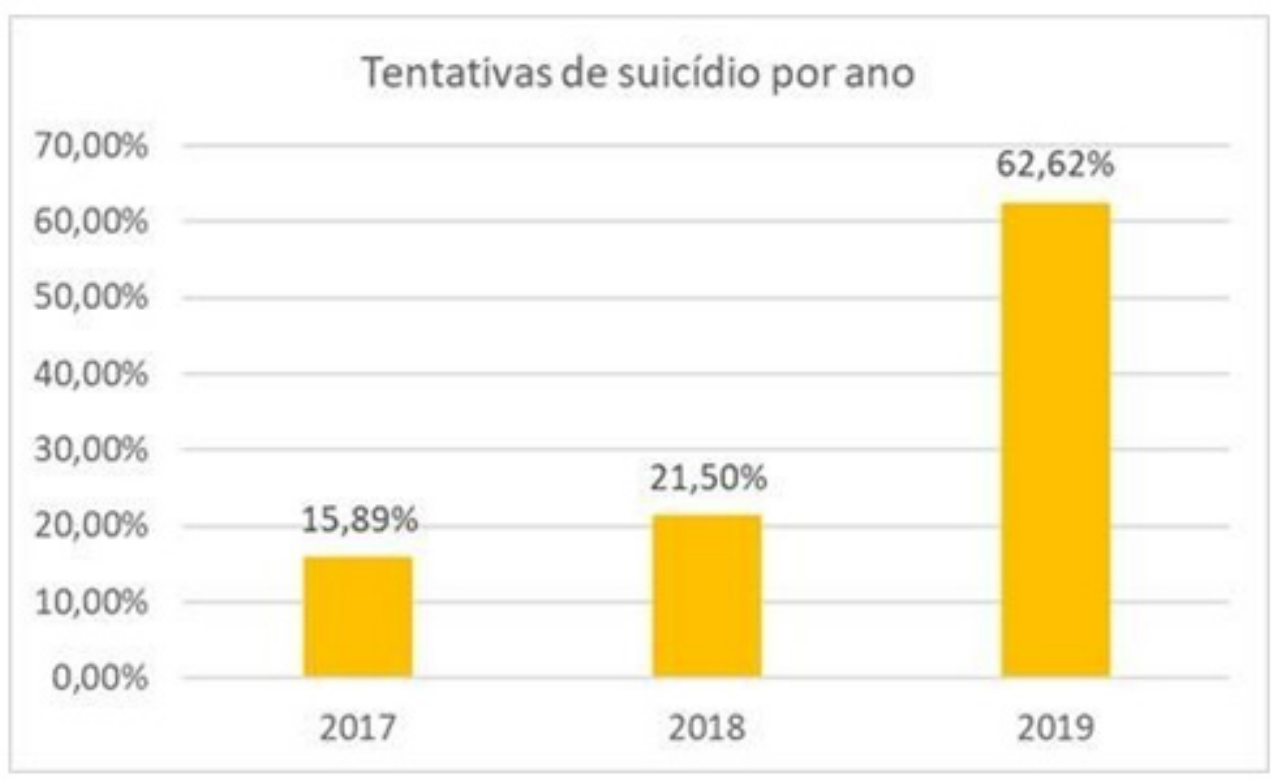

Quando se correlaciona tentativa de suicídio com o gênero do indivíduo, o sexo feminino mostrou risco $129 \%$ maior de apresentar tal motivação quando comparado ao masculino. Além disso, em relação a causa da intoxicação, 57,43\% dos indivíduos do sexo feminino que sofreram intoxicação foram em decorrência de tentativa de suicídio. Entretanto, quanto aos indivíduos do sexo masculino, 75\% das intoxicações não foram por tentativa de suicídio, sendo que 59,09\% de todas as exposições desse gênero ocorreram de forma acidental.

Quanto às substâncias utilizadas, aquelas classificadas como de fácil acesso tiveram um risco 56\% menor de serem usadas para tentativa de suicídio quando comparadas às de difícil acesso. Ademais, quando comparadas às substâncias não medicamentosas, as medicamentosas tiveram um risco $297 \%$ maior de serem usadas na tentativa de suicídio (Figura 6).

!' 
Figura 6 - Frequência dos medicamentos de fácil e de difícil acesso utilizados nas tentativas de suicídio.

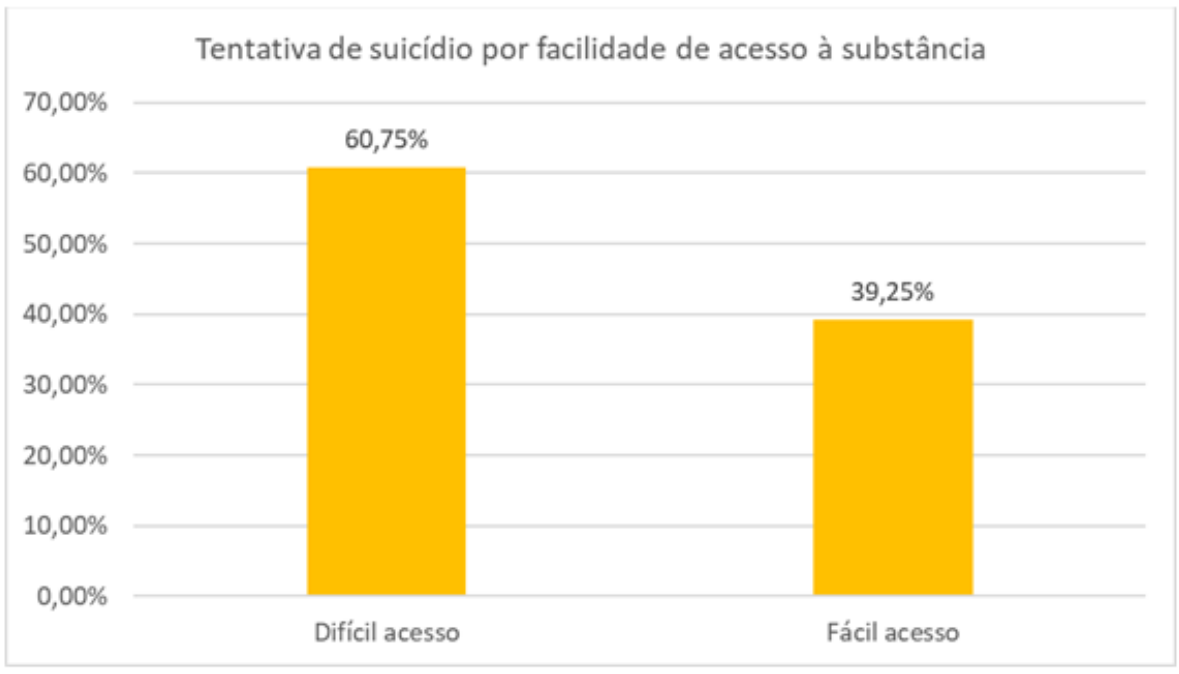

Considerando o local da exposição, pode-se verificar que a tentativa de suicídio foi $256 \%$ maior em residência quando comparado em outras localizações, como ambiente de trabalho, ambiente externo, escola/creche e serviços de saúde (Figura 7).

Figura 7 - Frequência dos locais de ocorrência das tentativas de suicídio.

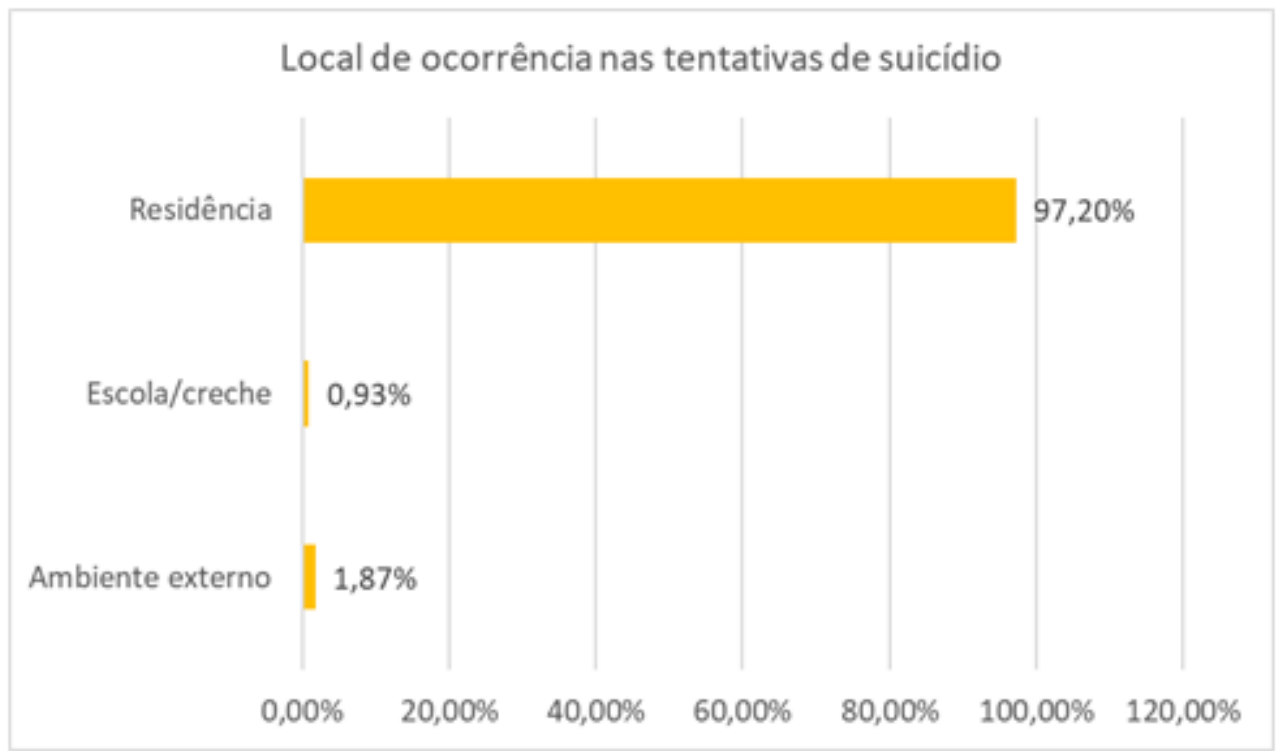

Ademais, o risco de tentativa de suicídio foi $29 \%$ menor no primeiro semestre quando comparado ao segundo semestre, sendo, então, o segundo semestre do ano considerado fator de risco para a tentativa de suicídio (Figura 8). Quanto às características populacionais e econômicas dos municípios em que ocorreram as intoxicações exógenas com o intuito de suicídio os dados foram irrelevantes quando analisados (Tabela 3). 
Figura 8 - Gráfico da frequência de tentativa de suicídio no primeiro e no segundo semestresdos anos de 2017 a 2019.

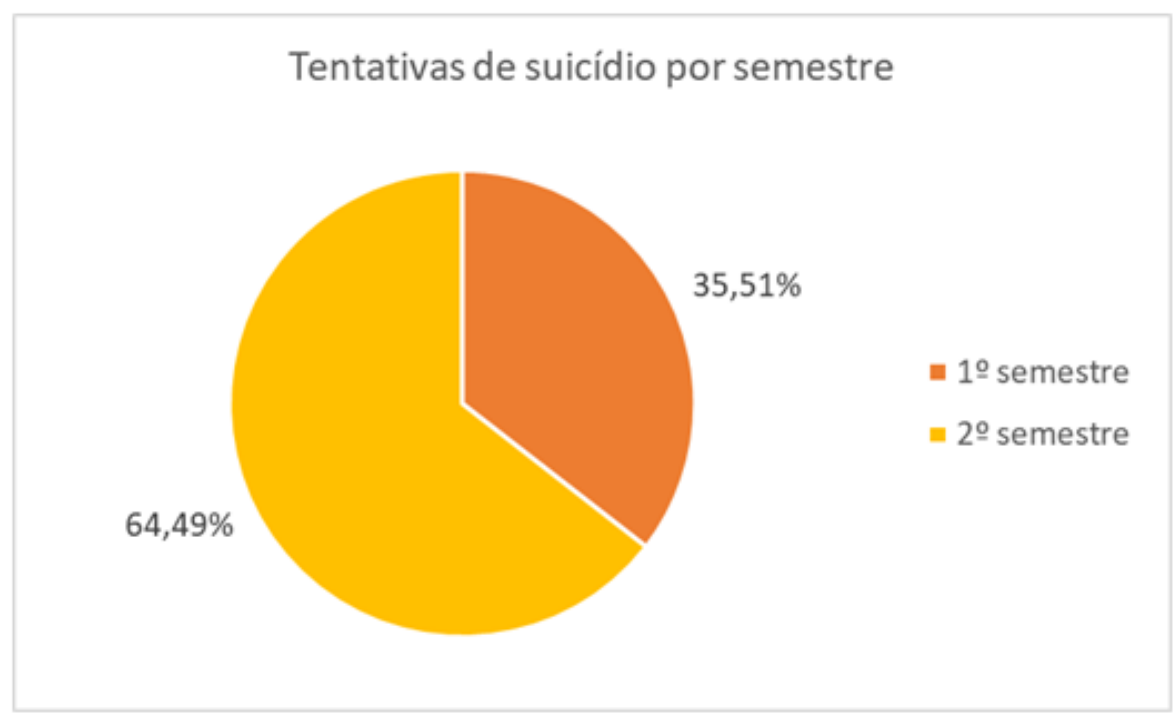

Tabela 3 - Análise bivariada de intoxicações exógenos decorrentes de tentativa de suicídio em indivíduos da faixa etária pediátrica no Mato Grosso, nos anos de 2017 e 2019.

\begin{tabular}{|c|c|c|c|c|}
\hline VARIÁVEIS & $\begin{array}{l}\text { TENTATIVA } \\
\text { DE SUICIDIO } \\
\end{array}$ & $\begin{array}{l}\text { OUTRAS } \\
\text { INTOXICAÇ̃̃ES }\end{array}$ & RP (IC95\%) & $\mathbf{p}$ \\
\hline \multicolumn{5}{|l|}{ SEXO } \\
\hline Feminino & $85(57,43 \%)$ & $63(42,57 \%)$ & $2,29(1,56-3,38)$ & \multirow[t]{2}{*}{$<0,001$} \\
\hline Masculino & $22(25,00 \%)$ & $66(75,00 \%)$ & 1,00 & \\
\hline \multicolumn{5}{|c|}{ SUBSTÂNCIADE } \\
\hline FÁCIL ACl & & & & \\
\hline Sim & $42(30,43 \%)$ & $96(69,57 \%)$ & $0,45(0,34-0,61)$ & \multirow[t]{2}{*}{$<0,001$} \\
\hline Não & $65(66,33 \%)$ & $33(33,67 \%)$ & 1,00 & \\
\hline \multicolumn{5}{|c|}{ MEDICAMENTO } \\
\hline $\operatorname{Sim}$ & $91(65,47 \%)$ & $48(34,53 \%)$ & $3,97(2,49-6,31)$ & \multirow{2}{*}{$<0,001$} \\
\hline Não & $16(16,49 \%)$ & $81(83,51 \%)$ & 1,00 & \\
\hline \multicolumn{5}{|c|}{ RESIDÊNCIA } \\
\hline $\operatorname{Sim}$ & $104(48,60 \%)$ & $110(51,40 \%)$ & $3,56(1,23-10,39)$ & \multirow{2}{*}{0,0017} \\
\hline Não & $3(13,64 \%)$ & $19(86,36 \%)$ & 1,00 & \\
\hline \multicolumn{5}{|l|}{ SEMESTRE } \\
\hline $1^{\circ}$ semestre & $38(36,89 \%)$ & $65(63,11 \%)$ & $0,71(0,52-0,96)$ & \multirow[t]{2}{*}{0,02} \\
\hline $2^{\circ}$ semestre & $69(51,88 \%)$ & $64(48,12 \%)$ & 1,00 & \\
\hline \multicolumn{5}{|c|}{ POPULAÇÃO } \\
\hline$>50$ mil hab & $59(48,36 \%)$ & $63(51,64 \%)$ & $1,14(0,86-1,52)$ & \multirow{2}{*}{0,93} \\
\hline$<50$ mil hab & $48(42,11 \%)$ & $66(57,89 \%)$ & 1,00 & \\
\hline \multicolumn{5}{|l|}{ POLO } \\
\hline ECONÔMI & & & & \\
\hline Sim & $76(46,91 \%)$ & $86(53,09 \%)$ & $1,11(0,82-1,53)$ & \multirow{2}{*}{0,47} \\
\hline Não & $31(41,89 \%)$ & $43(58,11 \%)$ & 1,00 & \\
\hline
\end{tabular}


1. Vale NB - Centenário da Raquianestesia Cirúrgica. Rev Bras Anestesiol, 1998; 48 507-520.

2. Choi PT, Galinski SE, Takeuchi L, et al. PDPH is a common complication of neuroaxial blockade in parturients: a meta-analysis of obstetrical studies. Can J Anaesth. 2003;50:460---9

3. Turnbull DK, Shepherd DB. Post-dural puncture headache: pathogenesis, prevention and treatment. Br J Anaesth. 2003;91:718---29.

5. Türkyilmaz EU, Eryilmaz NC, Güzey NA Moraloğlu O. Bilateral greater occipital nerve block for treatment of post-dural puncture headache after caesarean operations.

Brazilian Journal of Anesthesiology. 2016 SepOct; 66(5):445-450

7. Bogduk N. The clinical anatomy of the cervical dorsal rami. Spine (Phila Pa 1976). 1982 Jul-Aug; 7(4):319-30.

8. Anthony M. Cervicogenic headache: prevalence and response to local steroid therapy. Clin Exp Rheumatol. 2000;18:59-64.

\section{DISCUSSÃO}

De acordo com a literatura revisada neste artigo, a faixa etária pediátrica compõe o grupo de risco para intoxicações exógenas. Isto se justifica através da curiosidade inata de crianças menores de seis anos, com o hábito de explorar o ambiente e levar objetos à boca. Em contrapartida, crianças maiores e adolescentes estão mais envolvidos em intoxicações intencionais por medicamentos e drogas de abuso. ${ }^{1,3}$ Apesar da alta prevalência de intoxicações exógenas, eventos fatais são incomuns. ${ }^{5}$

Algumas características comportamentais e psicopatológicas frequentes relativas às crianças com intoxicação exógena acidental são a hiperatividade, o negativismo, os acessos de raiva, impulsividade e ousadia; ao considerar os aspectos parentais que são associados a estes eventos podemos citar a insatisfação conjugal, os sinais de sofrimento mental da mãe e a instabilidade emocional, a ansiedade e a depressão de qualquer um dos pais. Além destes agravantes, considerando que a criança está inserida em um círculo familiar, mesmo que disfuncional, é importante ressaltar a associação das intoxicações exógenas acidentais com as doenças familiares graves e a superlotação nas condições de vida, bem como a baixa renda familiar.6 Conforme visto neste estudo, ao se avaliar a faixa etária de 0 a 19 anos foi observado maior prevalência de intoxicações ocasionadas por medicamentos. ${ }^{1,2,7}$ No Brasil os fármacos compõem o ranking dos agentes intoxicantes nas estatísticas do Sistema Nacional de Informações Tóxico Farmacológica (SINITOX), que anualmente coordena e divulga o número de casos no país. Dados de 2017, por exemplo, demonstraram 20.637 casos registrados de intoxicação medicamentosa e aproximadamente $43 \%$ destes ocorrem na faixa etária citada acima. ${ }^{8}$

Um estudo realizado no Reino Unido, que investigou o número de envenenamento acidental em crianças, evidenciou que os benzodiazepínicos são responsáveis por 19\% das internações em unidade de terapia intensiva pediátrica (UTIP), os opioides $17 \%$ e antidepressivos tetra e tricíclicos 11\%.5 De acordo com os dados analisados no presente estudo observou-se que as classes dos benzodiazepínicos e antidepressivos também prevalecem como os 
1. Vale NB - Centenário da Raquianestesia Cirúrgica. Rev Bras Anestesiol, 1998; 48: 507-520.

2. Choi PT, Galinski SE, Takeuchi L, et al. PDPH is a common complication of neuroaxial blockade in parturients: a meta-analysis of obstetrical studies. Can J Anaesth. 2003;50:460---9.

3. Turnbull DK, Shepherd DB. Post-dural puncture headache: pathogenesis, prevention and treatment. Br J Anaesth. 2003;91:718---29.

7. Bogduk N. The clinical anatomy of the cervical dorsal rami. Spine (Phila Pa 1976). 1982 Jul-Aug; 7(4):319-30.

9. Binder WJ, Brin MF, Blitzer A, Schoenrock LD, Pogoda JM Otolaryngol Head Neck Surg. 2000 Dec; 123(6):669-76.

10. Ministério da Saúde (BR). Portaria n 264 de 17 de fevereiro de 2020. Altera a Portaria de Consolidação $n^{\circ}$ 4/GM/MS, de 28 de setembro de 2017, para incluir a doença de Chagas crônica, na Lista Nacional de Notificação Compulsória de doenças, agravos e eventos de saúde pública nos serviços de saúde públicos e privados em todo território nacional. Diário Oficial União. 19 fev 2020; Seção 1 .

11. Pfeiffer L, Cardon L. Da autoagressão velada ao suicídio. In: Fujiyoshi SM, editor. Tratado de pediatria: Sociedade Brasileira de Pediatria. 4. ed. Barueri: Manole; 2017. v. 1, p. 104-108 principais causadores de intoxicações medicamentosas em crianças e adolescentes no estado do Mato Grosso.

Ademais quando analisadas as substâncias não medicamentosas, concluiu-se que os agentes químicos como água sanitária e soda cáustica são os mais prevalentes nas intoxicações acidentais. Assim como nas intoxicações exógenas não intencionais, os agentes químicos também se destacam, o que demonstra a importância da prevenção de acidentes domésticos com esses produtos, visto que, foi identificado uma frequência majoritariamente maior desses eventos dentro das residências.

Consoante à literatura, neste artigo a tentativa de suicídio se destacou como a principal causa de intoxicação exógena na faixa etária pediátrica nos indivíduos de 15 a 19 anos e, na primeira infância, a causalidade dos eventos se dá por intoxicações exógenas acidentais. ${ }^{1,2,3,7,9}$ Entretanto, vale ressaltar o viés do erro de preenchimento das fichas de notificação, como é possível inferir pelo dado de duas tentativas de suicídio em menores de um ano presentes neste trabalho. Tal fato pode ser justificado pela falta de capacitação dos profissionais quanto aos métodos de preenchimento das fichas de notificação bem como, sobre a interpretação de termos como tentativa de suicídio, suicídio e intoxicação acidental.

De acordo com o SINITOX 8, faz-se necessário a correta alimentação dos bancos de dados para que os resultados encontrados não sejam subestimados. Dessa forma, destaca-se a importância do reconhecimento da natureza das fichas de notificação de intoxicações exógenas e as de violência interpessoal/autoprovocada (na qual se inclui as tentativas de suicídio), assim como sua frequência de repasse, pois apesar de ambas serem compulsórias, a primeira é de notificação semanal e a segunda é imediata (até $24 \mathrm{~h}) .{ }^{10}$

Segundo o estudo do Official Journal of the American Academy of Pediatrics (2020), o suicídio é a segunda maior causa de morte em indivíduos de 10-24 anos nos Estados Unidos e no mundo. $\mathrm{Na}$ faixa etária de cinco a 12 anos, esta é a quinta causa de morte, ademais entre indivíduos de 10 a 14 anos o suicídio tem aumentado progressivamente, chegando a ultrapassar o número de mortes por acidente de trânsito. ${ }^{11}$ Tais informações revelam a importância de se atentar à tendência mundial de aumento de óbitos por suicídio, visto que, no presente estudo, foi possível avaliar a crescente incidência 
11. Pfeiffer L, Cardon L. Da autoagressão velada ao suicídio. In: Fujiyoshi SM, editor. Tratado de pediatria: Sociedade Brasileira de Pediatria. 4. ed. Barueri: Manole; 2017. v. 1, p. 104-108.

12. Horowitz L, PhD, MPH, Tipton MV, BA, Pao $M, M D$. Primary and Secondary Prevention of Youth Suicide. Pediatrics Journal. 2020; 145:196-203.

13. Gordon MS, Melvin GA. Do antidepressants make children and adolescents suicidal. J of Paediatr Child Health. 2014 feb:847-854. de tentativas de suicídio no estado do Mato Grosso na faixa etária pediátrica, revelando o acréscimo de aproximadamente 294\% desses casos quando comparamos o ano de 2017 com 2019, não considerando o desfecho óbito, necessitando assim, de estudos que avaliem especificamente óbitos por suicídio neste estado para avaliar o crescimento deste desfecho nessa população.

A tentativa de suicídio na faixa etária pediátrica pode ser motivada por diversos fatores complexos, dentre eles grandes perdas, abandono, frustração e apatia, incertezas ou induções a distorção da sexualidade, transtornos psiquiátricos, bullying e abuso físico e sexual na infância. ${ }^{11}$ Frente ao histórico familiar, o desenvolvimento de psicopatologias em parte pode ser vinculado tanto a questões genéticas como de convivência. Dessa forma, através do tratamento das doenças psiquiátricas paternas, especificamente a depressão, observa-se redução dos sintomas depressivos e suicidas nos filhos promovendo melhora na saúde mental destes, como afirma Horowitz L. et al. ${ }^{12}$

Quando avaliadas exclusivamente crianças que fazem 0 uso de antidepressivos com as que não utilizam, como evidenciado na metanálise australiana "Do antidepressants make children and adolescents suicidal", observou-se que há um maior risco de comportamento e pensamento suicida naquelas em comparação a estas, especialmente no início do tratamento. Tal fato pode ser explicado por diversos mecanismos desencadeadores, dentre eles aumento da energia em detrimento da letargia e retardo psicomotor para execução de planos suicidas, piora da depressão e ansiedade, piora da insônia e aumento dos distúrbios da arquitetura do sono, mudança do eletroencefalograma com aumento da impulsividade. Ademais, tais medicamentos podem desencadear quadros de mania e hipomania quando instituído diagnóstico errôneo. ${ }^{13}$

A perspectiva de gênero em relação as tentativas de suicídio na faixa etária pediátrica são pouco estudadas. 0 que é possível observar através de diversos estudos é a prevalência do sexo feminino nestas tentativas, tal fato justifica-se por uma maior tendência entre elas de internalizar os sentimentos, predispondo a sintomas psicossomáticos, ansiosos e depressivos. No presente estudo, esse dado foi confirmado quando analisada a faixa etária dos 15 aos 19 anos $(p<0,05)$. Em contrapartida, meninos tendem a demonstrar sua dor na forma de 
7. Bogduk N. The clinical anatomy of the cervical dorsal rami. Spine (Phila Pa 1976). 1982 Jul-Aug; 7(4):319-30.

9. Binder WJ, Brin MF, Blitzer A, Schoenrock LD, Pogoda JM Otolaryngol Head Neck Surg. 2000 Dec; 123(6):669-76

14. Zambolim CM, Oliveira TP, Hoffmann AN, Vilela CEB, Neves D, Anjos FR, et al. Perfil das intoxicações exógenas em um hospital universitário. Rev med Minas Gerais. 2008;18(1):5- 10.

15. Fenning S, Geva K, Zalsman G, Weizman A, Fenning S, Apter A. Effect of gender on suicide attempters versus nonattempters in an adolescent inpatient unit. Rev

Comprehensive Psychiatry. 2005;46:90-97.

16. Heerlein A, Valeria C, Medina B. Seasonal Variation in Suicidal Death in Chile: Its

Relationship to Latitude. Psychopathology Journal. 2006;39:75-79.

17. Vieira LP, Santana VTP, Suchara EA. Caracterização de tentativas de suicídios por substâncias exógenas. Cad. Saúde Colet. 2015;23(2):118-123. alterações comportamentais, abuso de drogas e a cometer infrações legais, além de serem mais efetivos nas tentativas de suicídio; mesmo apresentando quadros depressivos de menor gravidade comparativamente. . $^{7,9,14,15}$

Por fim, quanto as diferenças sazonais, há muita divergência relativa à prevalência dentre os diversos autores. No Mato Grosso, durante o período estudado, concluiu-se com evidência estatística uma maior prevalência dos casos no segundo semestre do ano na faixa etária pediátrica. Apesar da informação consistente na literatura de que há relação entre a variação climática no decorrer das estações e a taxa de tentativa de suicídio, no Brasil, tal variação climática não é bem definida, tornando essa relação difícil de ser verificada. Segundo Heerlein A. et al. ${ }^{16}$, a maior incidência de suicídio no hemisfério sul ocorre em dezembro, porém segundo Vieira P.L. et al. ${ }^{17}$ o pico de incidência da tentativa de suicídio verificado em estudos com a população brasileira ocorre no Outono.

\section{CONCLUSÃO}

Analisando os dados obtidos pelo estudo, foi observado no estado de Mato Grosso o aumento do número de casos de intoxicações exógenas na faixa etária pediátrica nos anos de 2017 a 2019, principalmente pela utilização de medicamentos. Associado a isso, quando se comparam as circunstâncias de ocorrência, notou-se que a tentativa de suicídio se sobressai na faixa etária dos 15 aos 19 anos e, em contrapartida, as causas acidentais predominam nos menores de 10 anos.

Conforme revisto no estudo, as principais medicações envolvidas são os benzodiazepínicos e os antidepressivos tanto nos casos intencionais quanto em não intencionais. Nas ocorrências com substâncias não medicamentosas, os agentes químicos como água sanitária e soda cáustica, se destacam como agente tóxico.

Em consonância com a literatura, as intoxicações consequentes de tentativa de suicídio, predominam nos adolescentes e estão ligados a diversos fatores ambientais, psicossociais e genéticos, como histórico familiar, transtornos psiquiátricos prévios e frustrações pessoais. Por fim, observou-se a prevalência do sexo feminino nas tentativas 
de suicídio quando comparado ao sexo masculino, por fatores ainda não explicitados nos diversos estudos.

\section{REFERÊNCIAS}

1. Paulis M. Intoxicações exógenas agudas. In: Fujiyoshi SM, editor. Tratado de pediatria: Sociedade Brasileira de Pediatria. 4. ed. Barueri: Manole;2017. v. 1, p. 223-229.

2. Loureiro I, Tavares A, Costa S, Carvalho IP. Intoxicações. In: Carvalho IP, Mansilha HF, Matos P, Almeida R, coordenadores. Urgência Pediátrica Integrada do Porto. 1 ed. 2010. p. 605-612.

3. Silva TJ, Oliveira VB. Intoxicação medicamentosa infantil no Paraná. Rev Visão Acadêmica. 2018;19(1):51-61.

4. Sadock BJ, Sadock VA, Ruiz P. Compêndio de Psiquiatria. Porto Alegre: Artmed; 2017. Medicina Psiquiátrica de Emergência; p. 763-774.

5. Anderson M, Hawkins L, Eddleston M, Thompson JP, Vale JA, Thomas SHL. Severe and fatal pharmaceutical poisoning in Young children in the UK. Arch Dis Child. 2016;101:653- 656.

6. Katrivanou A, Lekka NP, Baratis S. Psychopathology and behavioural trends of children with accidental poisoning. J Psychosom Res.2004;57:95-101.

7. Oliveira FFS, Suchara EA.Perfil epidemiológico das intoxicações exógenas em crianças e adolescentes em município do Mato Grosso. Rev Paul Pediatr. 2014;32(4):299-305.

8. SINITOX: Sistema Nacional de Informações Tóxico-Farmacológicas [internet]. Rio de Janeiro: Fiocruz. Casos registrados de Intoxicação Humana por agente toxico e faixa etária. Brasil, 2017. Rio de Janeiro: Fiocruz. [acesso em 01 de set 2020]. Disponível em: http://www.fiocruz.br/sinitox

9. Alcântara DA, Vieira UES, Albuquerque VLM. Intoxicação medicamentosa em criança. Rev Bras Promoç Saúde. 2003;16:10-16.

10. Ministério da Saúde (BR). Portaria $n^{\circ}$ 264, de 17 de fevereiro de 2020. Altera a Portaria de Consolidação $n^{\circ}$ 4/GM/MS, de 28 de setembro de 2017, para incluir a doença de Chagas crônica, na Lista Nacional de Notificação Compulsória de doenças, agravos e eventos de saúde pública nos serviços de saúde públicos e privados em todo território nacional. Diário Oficial União. 19 fev 2020; Seção 1. 
11.Pfeiffer L, Cardon L. Da autoagressão velada ao suicídio. In: Fujiyoshi SM, editor. Tratado de pediatria: Sociedade Brasileira de Pediatria. 4. ed. Barueri: Manole; 2017.v. 1, p. 104-108.

12. Horowitz L, PhD, MPH, Tipton MV, BA, Pao M, MD. Primary and Secondary Prevention of Youth Suicide. Pediatrics Journal. 2020; 145:196-203.

13. Gordon MS, Melvin GA. Do antidepressants make children and adolescents suicidal. J of Paediatr Child Health. 2014 feb:847-854.

14.Zambolim CM, Oliveira TP, Hoffmann AN, Vilela CEB, Neves D, Anjos FR, et al. Perfil das intoxicações exógenas em um hospital universitário. Rev med Minas Gerais. 2008;18(1):5- 10.

15. Fenning S, Geva K,Zalsman G, Weizman A, Fenning S, Apter A. Effect of gender on suicide attempters versus nonattempters in an adolescent inpatient unit. Rev Comprehensive Psychiatry. 2005;46:90-97.

16. Heerlein A, Valeria C, Medina B. Seasonal Variation in Suicidal Death in Chile: Its Relationship to Latitude. Psychopathology Journal. 2006;39:75-79.

17. Vieira LP, Santana VTP, Suchara EA. Caracterização de tentativas de suicídios por substâncias exógenas. Cad. Saúde Colet. 2015;23(2):118-123. 